Revue Sciences/Lettres

Sciences / LetTres

2 | 2014

Épistémologies digitales des sciences humaines et sociales

\title{
Le réseau et ses outils comme lieu de raffinement
} du livre

\section{Chloé Girard}

\section{(2) OpenEdition \\ Journals}

Édition électronique

URL : http://journals.openedition.org/rsl/526

DOI : $10.4000 /$ rsl. 526

ISSN : 2271-6246

Éditeur

Éditions Rue d'Ulm

Référence électronique

Chloé Girard, "Le réseau et ses outils comme lieu de raffinement du livre », Revue Sciences/Lettres [En ligne], 2 | 2014, mis en ligne le 07 octobre 2013, consulté le 19 avril 2019. URL : http://

journals.openedition.org/rsl/526; DOI : 10.4000/rsl.526

Ce document a été généré automatiquement le 19 avril 2019

(c) Revue Sciences/Lettres 


\title{
Le réseau et ses outils comme lieu de raffinement du livre
}

\author{
Chloé Girard
}

\section{La forme du livre et la « fin de l'histoire »}

1 Le passage à l'édition mixte, papier et numérique, a induit puis dépassé différentes craintes : celles de la fin du livre, disparaissant avec son support; celle de la fin de son histoire, avec la transition vers un nouvel objet, le Read/Write Book ${ }^{1}$, qui ne distingue plus livre/texte/document, blog, journal, forum ou encyclopédie collaborative; ou celle de la fin de l'Histoire elle-même, avec le livre liquide, si l'on considère à l'instar de Michel Foucault que l'Histoire est « une certaine manière pour une société de donner statut et élaboration à une masse documentaire dont elle ne se sépare pas »².

2 Le livre électronique, applicatif ou non, a acquis finalement sa légitimité et semble conserver sa place dans l'ordre des discours, aux côtés de nouvelles formes éditoriales numériques : blogs, wikis, catalogues personnalisés... Nous avons accepté que le livre soit à deux dimensions tout en maintenant sa distinction en exemplaire détaché, unité formelle censée le distinguer notamment des contenus en ligne. Ceux-ci sont supposés ne pas avoir de limites, le web est donc le lieu d'autres formes de discours.

3 Cette dichotomie livre/contenus web demande d'être questionnée car elle suppose et peut ancrer une exclusion du livre d'un lieu sans pareil d'exploitation et d'analyse des savoirs. En tant que co-conceptrice d'un logiciel d'édition mixte (électronique/papier), j'ai pu prendre la mesure du potentiel comme des limites, le cas échéant, de ce que l'on nomme format électronique, cet assemblage dynamique de fichiers de texte, de scripts informatiques, de systèmes d'exploitation et de moteurs de rendu (la conception du format comme une entité statique a vécu). Et en tant que responsable de fabrication pour un éditeur de sciences littéraires, je dois envisager la fabrication de livres qui sont des objets de lecture simple mais aussi des outils de travail. Je tiens donc à leur définition précise, à leur solidité, autant qu'à leur aspect de livre-service. 

aussi bien au livre de s'y transposer qu'à d'autres genres éditoriaux de s'y spécifier. Le web n'est pas le lieu de la disparition du livre au profit de formes concurrentes mais bien celui du raffinement de l'ordre des discours et de la spéciation du livre en tant que démarche spécifique. Des discours qui n'avaient de livre que la forme trouvent enfin les outils d'édition et supports de publication convenant à leur objet tandis qu'en retour le livre se distingue de mieux en mieux. Nous distinguons d'autant mieux un essai politique que nous pouvons le comparer au blog d'actualité dont la rédaction, dans son ensemble, est par principe dénuée de plan et la présentation chronologique, y compris à l'intérieur des «catégories». Nous distinguons également de mieux en mieux un livre de cuisine, sous-tendu par un principe d'auteur fort, d'un catalogue contextuel de recettes (ce n'est pas péjoratif) bâti sur des critères tels que "avec tel ingrédient» et "pour un coût maximum de tant ». Et nous faisons ces distinctions quand bien même les deux contenus seraient inscrits dans le réseau, seraient des « contenus web».

Accepter le livre comme élément de l'ordre des discours indépendant de son inscription matérielle permettrait de façonner des outils d'édition et de publication au plus près de ses exigences, plutôt que de subir les effets induits par un soi-disant «système d'information électronique» ou de l'en voir exclu. Qu'importe, sur le plan épistémologique, que le livre de cuisine électronique se vende ou non face à l'observation de la spéciation de différentes formes « littéraires » au moyen d'outils plus nuancés que le papier et la reliure?

6 Pour sortir de cette fausse antinomie contenu web/livre et autoriser le second à entrer intelligemment dans le réseau il est nécessaire de finir de s'affranchir de la définition du livre par la forme ou l'identification immédiate d'une unité.

\section{Les procédures de l'ordre du discours chez Foucault Leur pertinence et leur respect au moyen des technologies d'édition numériques}

7 À propos des unités «livre » et «œuvres », en apparence celles qui «s'imposent de la façon la plus immédiate » quand on analyse le discours et le savoir, Michel Foucault écrit en 1969 :

«[...] l'unité matérielle du volume n'est-elle pas une unité faible, accessoire, au regard de l'unité discursive à laquelle il donne support? Mais cette unité discursive, à son tour, est-elle homogène et uniformément applicable ? Un roman de Stendhal ou un roman de Dostoïevski ne s'individualisent pas comme ceux de La Comédie humaine; [...] C'est que les marges d'un livre ne sont jamais nettes ni rigoureusement tranchées : par-delà le titre, les premières lignes et le point final, par-delà sa configuration interne et la forme qui l'autonomise, il est pris dans un système de renvois à d'autres livres, d'autres textes, d'autres phrases : nœud dans un réseau. [...] Le livre a beau se donner comme un objet qu'on a sous la main; il a beau se recroqueviller en ce petit parallélépipède qui l'enferme: son unité est variable et relative. ${ }^{3}$ »

8 Alors qu'en 1969 le livre n'existe encore que sous sa forme imprimée, celle-ci est pour Foucault un simple mode d'autonomisation et le livre n'a pas d'unité définie. L'unité matérielle du livre est une unité faible. La forme ne dit rien de l'unité discursive, unité 
bien plus forte, quoique le livre soit toujours mieux défini en tant que «nœud dans un réseau ».

Le livre n'a pas de réelle unité mais une place dans le discours. C'est cette place et ce qui la caractérise qu'il nous faut définir et, si elle nous paraît encore nécessaire aujourd'hui, dont nous devons voir si elle peut être reconduite dans le réseau.

Dans son discours inaugural prononcé lors de son entrée au Collège de France en 1970 et intitulé précisément "L'ordre du discours » Foucault définit celui-ci comme un ensemble de procédures qui ont pour rôle de contrôler et de délimiter le discours, « d'en conjurer les pouvoirs et les dangers, d'en maitriser l'événement aléatoire, d'en esquiver la lourde, la redoutable matérialité $»^{4}$.

11 Le positionnement des curseurs correspondant à chacune de ces procédures assure un ordre, un contrôle du discours et définit des formes éditoriales parmi lesquelles le livre et ses genres. Les outils web s'opposent-ils à la maîtrise fine de chacune de ces procédures ?

\section{Le fou et le sage}

12 Il y a d'abord des procédures externes aux énoncés, qui sont des principes d'interdit, d'opposition entre le vrai et le faux, entre raison et folie. Elles ne dépendent cependant pas des outils de production et de publication du discours et ne seront donc pas l'objet de ce texte. Avec une réserve cependant. Car, si les notions de fou et de sage ne dépendent pas du papier ou de l'électronique ce n'est pas forcément vrai de la question de la raréfaction des sujets parlants que nous verrons plus loin. Or l'un peut avoir un effet retour sur l'autre. Si le sujet parlant est moins rare, du fait de la technique, peut-être donne-t-il aussi plus l'occasion au fou de parler, ce qui en fait finalement quelqu'un de moins fou (le fou étant celui dont le discours ne peut pas être accepté) et modifie d'autant l'ordre du discours.

\section{Les procédures internes}

Les trois procédures internes, principe d'auteur, commentaire et discipline, sont par contre directement liées au mode de production du discours.

\section{Le principe d'auteur}

14 Le principe d'auteur (ou argument) est un principe de groupement du discours qui soustend l'ouvrage. Peut-il rester explicite dans l'édition en réseau, sachant que ce principe peut être l'individu lui-même, un institut signataire de l'ouvrage ou une thèse, un argument?

Prenons d'abord l'identification des auteurs d'un livre, la question de la signature. Dans le cas le plus « dangereux » en apparence de la rédaction en ligne on sait que la distinction et le contrôle des auteurs passe par une simple gestion de niveaux d'accès. Ce contrôle peut être très fort comme totalement libre. Il s'agit d'une décision éditoriale et non d'un effet induit par le réseau.

L'outil peut-il interférer avec la thèse, l'argument qui sous-tend la cohérence de l'ouvrage quand ce n'est pas la signature à elle seule qui la lui donne ? On peut imaginer plusieurs contraintes à la liberté de l'auteur ou de l'éditeur de maîtriser son argument et de le 
signifier au lecteur : un outil interférant directement dans la rédaction en limitant par exemple la modification du plan à un certain stade d'avancement du travail ; ou un outil empêchant la définition d'une collection, catégorie qui regroupe des ouvrages sous un tronc commun qui circonscrit l'argumentaire. Nous pouvons penser également à des outils non seulement de production mais aussi de lecture qui ne pourraient pas rendre une partie de l'œuvre: affichage de contenus multimédias, expression de différents niveaux de lecture, fichier trop lourd pour être ouvert, format non interopérable d'un appareil de lecture à un autre, etc.

Il faut s'assurer d'offrir aux auteurs des outils au moins aussi souples que des feuilles volantes. Mais aussi que les outils de lecture pourront rendre justice à la souplesse de production. L'édition électronique en réseau ouvre par exemple la liberté éditoriale du multimédia et des contenus dynamiques, contextuels. Or ce sont des libertés dangereuses quant au principe d'auteur, qui risque de perdre de sa force d'un format ou d'un support de lecture à l'autre. Surtout si l'on envisage encore l'impression papier parmi les formats de lecture.

Mais est-ce que le support de lecture papier n'impose pas déjà des contraintes aux auteurs? Le papier impose des contraintes mécaniques (le volume de 2000 pages impossible à manipuler), psychologiques (un livre de 1500 pages susceptible de décourager les lecteurs et obligeant à resserrer sa thèse), commerciales (le coût de fabrication et de distribution d'un ouvrage volumineux).

Les caractéristiques du papier imposaient aux auteurs certaines restrictions. Les technologies actuelles d'édition et de publication en présentent de nouvelles (processeurs, moteurs de rendu...) tout en en résolvant certaines. Le respect du principe d'auteur n'est donc pas plus menacé a priori dans l'édition en réseau que dans l'édition papier.

\section{Le commentaire : distinction texte premier/texte second}

20 La notion de commentaire pose la question de la distinction entre texte premier, le texte "lui-même", et texte second, le commentaire. Il faut donc déjà pouvoir définir le premier.

21 Cela implique de le finir et de pouvoir l'identifier. Il faut pour cela pouvoir faire acte d'édition, de clôture du texte, de façon aussi forte que lorsque l'on envoie un bon à tirer à l'impression. Or cela consiste tout simplement à fermer l'accès des auteurs au texte. Aux éditeurs de garantir cette clôture afin d'éviter la citation relative à une date de consultation. Cela relève uniquement de leur responsabilité et non de la technique qui nous offre toute latitude possible sur ce plan jusqu'au chiffrement des fichiers permettant de vérifier que le contenu, l'édition, est bien original.

La difficulté avec l'édition électronique consiste ensuite à donner une identification au texte que tous les lecteurs pourront retrouver quelle que soit la forme qu'ils ont entre les mains. Car le commentaire y fait référence, et pas forcément au texte dans son ensemble mais aussi à l'un de ses extraits. Sachant que le texte publié en réseau peut s'abstraire de toute notion de page il faut d'une part envisager d'autres modes de référencement des contenus puis s'assurer qu'ils seront bien identifiables d'une forme à l'autre, du papier aux différentes formes électroniques. 
donc à nouveau la responsabilité des auteurs et des éditeurs au moment de la rédaction de l'ouvrage: chacun doit comprendre ces notions de rendu, de formats, d'interopérabilité mais aussi de serveur et envisager en conséquence d'inclure dans ses textes plus ou moins de contenus multisupports, multiformats et multilecteurs avec la complexification que cela induit dans l'identification univoque du texte premier. le commentaire le soit également, qu'il ne puisse y avoir confusion. Car d'une part le commentaire ne doit pas interférer avec le texte premier et d'autre part on doit pouvoir commenter un commentaire, sans pour autant le modifier. Le commentaire, qu'il se trouve en marge du texte premier ou dans un post sur le blog d'un lecteur, doit donc être aussi solide et identifiable que le texte premier. Il doit pouvoir devenir à son tour texte premier pour d'autres commentaires.

30 La question revient donc toujours à cette problématique de définition et d'identification des textes, premier ou commentaire, non seulement dans le réseau mais d'une forme à 
l'autre. L'absence de ce paramètre empêcherait le passage dans le réseau du livre entendu comme texte fixé, écrit ou composé par un auteur ou un éditeur selon une règle identifiable, c'est-à-dire avec un principe d'auteur fort (une idée, thèse, argumentaire, histoire, etc.). Nous aurions effectivement un texte éternellement réinscriptible, liquide, impossible à commenter et par conséquent inexistant.

«Le commentaire conjure le hasard du discours en lui faisant la part: il permet bien de dire autre chose que le texte même, mais à condition que ce soit ce texte même qui soit dit et en quelque sorte accompli.). ${ }^{5}$ ”

\section{La discipline}

31 La possibilité de répondre aux exigences et méthodes d'une discipline donnée est pour Foucault l'un des piliers de l'ordre des discours. Cela implique d'avoir les moyens d'écrire des équations mathématiques aussi bien que de distinguer des gloses selon que l'on est physicien ou philologue. Cela implique de pouvoir rédiger un glossaire aussi bien qu'une épigraphe. De distinguer des références à d'autres ouvrages comme de dessiner des tableaux complexes. Tous les codes qui fondent les écrits dans une discipline ou une autre doivent pouvoir être rendus, et ceci, encore une fois, quel que soit le format de sortie, avec la limite des compétences de chaque format.

Les outils réseau offrent précisément une batterie d'applications permettant d'y répondre de façon de plus en plus précise. Au point que certaines formes éditoriales y trouvent d'ailleurs matière à libérer leur "discipline» de la contrainte pas toujours fertile du papier : formes éditoriales basées sur l'actualité (quotidiens aujourd'hui mis à jour (!) en continu et pour lesquels il va nous falloir bientôt une autre appellation, mais aussi encyclopédies au volume important, catalogues offrant de nombreux critères d'entrée, etc.), sur la personnalisation (mes voyages, mes recettes... dont le lectorat ne pouvait justifier les frais d'une fabrication papier). On peut également citer l'édition scolaire et le raffinement des publications selon les niveaux des élèves, la mise à disposition conditionnelle des corrections, etc.

Des trois principes internes de l'ordre des discours (principe d'auteur, commentaire et discipline) aucun n'est donc logiquement impossible à conserver et à manipuler dans le passage à l'édition en réseau. Aucun n'est intrinsèquement lié au papier ni à une unité matérielle. Certains posent quelques défis techniques. Les trois demandent des restrictions volontaires aux auteurs/éditeurs, non pas par rapport à leurs anciennes habitudes mais par rapport aux nouvelles possibilités que les technologies en réseau leur offrent. Ces restrictions ne tiennent qu'à la limite de l'interopérabilité entre formats de données et supports de lecture. Les éditeurs doivent en conséquence être conscients de ces questions comme ils connaissaient les papiers et qualités de brochage. Ni plus ni moins.

\section{Raréfaction du sujet parlant}

Le principe de raréfaction des sujets parlants est sans doute le principe le plus remis en question par les outils réseaux. Si tout un chacun pouvait jusqu'à aujourd'hui rédiger quelque énoncé que ce soit et le publier sur papier en se passant de prescripteur (éditeur), il se trouvait néanmoins plus ou moins exclu des cercles autorisés et par conséquent d'une réelle diffusion. Or être sujet parlant signifie être lu. 
si tant est qu'on la considère comme une menace, ce jugement n'étant pas notre objet ici. Un bon ou un mauvais livre passé inaperçu peut aujourd'hui plus facilement être repêché. La notion de livre épuisé va disparaître et tout auteur pourra envisager d'accéder à la parole, même tardivement (ou s'inquiéter de la pérennité d'un écrit qu'il aurait préféré voir disparaître).

39 La structuration des contenus, quel que soit le schéma de structuration choisi, aura bien sûr un impact sur cet accès à la parole. Elle permettra aussi de raffiner la recherche et d'exhumer à l'occasion des ouvrages un temps négligés. Sur le plan technique la raréfaction des sujets parlants dépendra donc aussi de la structuration des contenus et de leur accessibilité aux outils web de recherche et d'indexation. D'où peut-être une certaine réticence à envisager le livre html intégré au réseau par rapport à une forme plus empaquetée, moins accessible aux outils de recherche et donc à la notoriété plus maîtrisable. Le livre doit pour rester un élément de discours auquel seules certaines personnes habilitées peuvent accéder. Ouvrir à chacun, théoriquement, la maîtrise du 
discours livre qui fonde en partie notre humanisme ${ }^{6}$ et constitue un pouvoir, est encore largement subversif.

\section{Le raffinement de l'ordre des discours grâce aux outils d'édition réseau}

Le web et la production comme l'exploitation dans le réseau ne s'opposent techniquement en aucun cas au respect des procédures du discours livre. Rien ne s'oppose, ni logiquement ni techniquement, à la distinction des auteurs, comme à leur confusion, aux commentaires comme au texte premier, ni à la subtilité que demandent les disciplines. Il semble également peu probable que tous les livres écrits et mis à disposition sur le réseau se confondent en intérêt et qualité aux yeux des lecteurs, ni même que ceuxci se passent de prescripteurs qui régulent par leur temps de lecture, leur travail éditorial ou leur critique un flot ininterrompu de publications.

La marge de manœuvre est totale et par conséquent aucun genre de discours écrit n'est menacé intrinsèquement par le réseau et ses outils. Nous assistons bien plutôt à un raffinement des formes que ne permettait pas la forme papier.

L'observation de cette nuance implique qu'on ne peut opposer contenus web et livre. Les deux catégories ne s'excluent en aucun cas. Et la concurrence que l'on semble percevoir n'est que le reflet d'un manque de livres dans le réseau : un essai explicite dont l'on peut copier/coller des extraits à citer avec une référence claire et pérenne, au paragraphe ou au signe près, identique d'une forme de publication à l'autre; un roman tout aussi identifiable et « solide », accessible depuis n'importe quel appareil de lecture; un livre de cuisine à la thèse forte, ayant tout à perdre à être personnalisé, une édition de texte ancien pouvant être interfacée avec des outils d'analyse lexicographiques et bibliographiques, etc. En l'absence de tels ouvrages facilement accessibles et exploitables dans le réseau, au moyen de logiciels universels (navigateurs et leurs extensions), peutêtre d'autres formes de discours font-elles office de succédanés acceptables? Mais c'est une victoire par abandon du terrain. Or, avec sa profondeur de champ technologique, le web est le terrain le plus fertile à l'avenir, pour ce qui concerne l'exploitation fine des savoir aussi bien que pour les opportunités commerciales.

\section{L'évolution des espèces éditoriales}

Le livre et les différents genres littéraires, l'ordre qu'ils confèrent au discours, sont des catégories complexes qui ne vivent aucune révolution aujourd'hui, encore moins de fin, de rupture ou de destruction. Le livre n'est pas plus en danger dans le web que les autres formes éditoriales mais bien plutôt en cours de spéciation, au sens de définition progressive d'une espèce dans un processus qui en voit naître de nouvelles.

De nouveaux outils, dont nous maîtrisons chaque ligne de code si nous nous y impliquons, raffinent des catégories qui rassemblaient jusque-là tant bien que mal des discours très éloignés les uns des autres et dont l'objet (personnalisation, actualisation, etc.) trouve finalement son expression pleine.

Nous avons de ce fait le devoir de comprendre le web et ses outils, sa profondeur plutôt que sa soi-disant réduction à deux dimensions. Aucun «système d'information 
électronique » n'existe que nous ne maîtrisons pas, ou ne pouvons maîtriser. Il nous revient donc, à nous éditeurs, auteurs, et concepteurs d'outils d'édition, de déterminer quelles formes éditoriales nous intéressent et de décider ensemble comment techniquement en rendre compte plutôt que d'envisager telle ou telle fatale concurrence entre elles.

\section{NOTES}

1. Marin Dacos (dir.), Read/Write Book. Le livre inscriptible, Marseille, Cléo, "Coll. Édition électronique ", 2010.

2. Michel Foucault, L'Archéologie du savoir, Paris, Gallimard, 1969.

3. Foucault, 1969, op. cit.

4. Michel Foucault, L'Ordre du discours, leçon inaugurale, Collège de France, Paris, Flammarion, 1971, p. 11.

5. Foucault, 1969, op. cit.

6. Peter Sloterdijk, Règles pour le parc humain, Paris, Mille et une nuits, «La petite collection », 2000.

\section{RÉSUMÉS}

Contenu web et livre sont souvent opposés l'un à l'autre comme deux formes de discours s'excluant mutuellement. Le livre comme ordre de discours ne peut-il cependant s'intégrer au réseau sans s'y dissoudre ? Nous voyons ici en suivant la trame des procédures de l'ordre du discours définis par Michel Foucault que les outils réseau sont propres non seulement à leur rendre justice mais plus encore à les raffiner. Le web n'est pas le lieu de la disparition du livre ou de quelque type de discours mais au contraire de leur spéciation la plus fine, là ou le papier les contraignait sous une appellation commune de livre dont ils n'avaient parfois que l'inévitable forme. La condition de ce raffinement du livre dans le réseau reste cependant la prise en main par les auteurs et éditeurs de ces techniques ainsi que leur implication dans la conception de ces nouveaux outils métier.

Web content and books are often presented as mutually exclusive forms of discourse. Can not the book, as a category of discourse be integrated into the web without falling apart? By following the framework of the procedures of categories of discourse defined by Michel Foucault, we can see that web tools will not only do these procedures justice but will also refine them. Rather than disappearing on the web, the various types of discourse actually achieve their finest speciation. Paper constrains them under the common name book, even if they only have the form of a book. The level of refinement that books will attain on the web, however, will depend on the authors and publishers of these techniques as well as their implication in the conception of new tools of the trade. 
INDEX

Mots-clés : livre, web, ordre du discours, logiciel, Michel Foucault, principe d'auteur, discipline, spéciation

Keywords : book, forms of discours, software, author principle, scientific discipline, speciation process

\section{AUTEUR}

\section{CHLOÉ GIRARD}

Maître de conférences associé à l'Enssib, responsable de fabrication numérique aux éditions Droz. 